\title{
Optimal selection of control structure using a steady-state inversely controlled process model
}

\author{
Mahdi Sharifzadeh ${ }^{\mathrm{a}, *}$, Nina F. Thornhill ${ }^{\mathrm{a}}$ \\ ${ }^{a}$ Centre for Process System Engineering (CPSE), Department of Chemical Engineering, Imperial College London, \\ *Email: mahdi@imperial.ac.uk. Address: Department of Chemical Engineering, Imperial College London, South \\ Kensington Campus, London SW7 2AZ.
}

\begin{abstract}
The profitability of chemical processes strongly depends on their control systems. The design of a control system involves selection of controlled and manipulated variables, known as control structure selection. Systematic generation and screening alternative control structures requires optimization. However, the size of such an optimization problem is much larger when candidate controllers and their parameters are included and it rapidly becomes intractable. This paper presents a novel optimization framework using the notion of perfect control, which disentangles the complexities of the controllers. This framework reduces the complexity of the problem while ensuring controllability. In addition, the optimization framework has a goal-driven multi-objective function and requires only a steady-state inverse process model. Since dynamic degrees of freedom do not appear in a steady-state analysis, engineering insights are employed for developing the inventory control systems. The proposed optimization framework was demonstrated in a case study of an industrial distillation train.
\end{abstract}

Keywords: control structure selection, multi-objective optimization, inversely controlled process model, perfect control. 


\section{Introduction}

Chemical process designers almost always intend to maximize the profits, while respecting environmental and safety regulations (Christofides and Davis 2007). However, their economic intentions often drive the chemical processes to operate towards their operational boundaries, such as maximization of the throughput, or minimization of wastes (Narraway and Perkins 1993; Edgar 2004). The main task of a control system is realization of the process design objectives in the presence of uncertainties. Uncertainties during process operation may cause the control objectives that are set in the design stage to deteriorate. The source of these uncertainties may be changes in the quality or the quantity of the feedstock or the products specifications, significant changes in the ambient conditions, fluctuations in the utility supplies or changes in the economic parameters. The purpose of the control system is to compensate for such effects.

Plant-wide Control Structure Selection concerns the decisions regarding selection of controlled variables (CVs), manipulated variables (MVs), and controllers which receive the measurements of the controlled variables and adjust the manipulated variables. A recent review of the methods for control structure selection is presented by Vasudevan et al. (2009). However, industrial practice still relies on insights and heuristics that have been developed and understood through engineering practice (Downs and Skogestad 2009). One way forward for more automated plant-wide control structure selection of large-scale chemical processes is to use an optimization framework. The main challenge, however, is that the number of combinations of alternative designs increases sharply with the size of the process and becomes intractable. This suggests that the control designer should employ a systematic method to reduce problem complexity.

This paper presents a new optimization framework for optimal selection of control structures. In this framework, the concept of perfect control is included in the optimization formulation. The proposed framework distinguishes between perfect control and optimal control. The perfect control is the best performance that a given control structure can achieve, whereas the optimal control is the performance of the best performing perfect control structure. Criteria are introduced and discussed in Section 2.4 that can be used to screen between alternative perfect control structures. The aim of the proposed method is different from flexibility analysis (Grossmann and Floudas 1987) in which the feasible region of process operation is quantified based on active constraints. Flexibility analysis does not have any implication for control performance because it does not make any assumption about the control structure. The proposed method for optimal control structure selection is a sequential design strategy. In a sequential design, the process is designed first and the feasible operating region is already identified. Then, the method for control structure selection aims at specifying the best control strategy and selecting the best control structure for that process. Examples of sequential designs are retrofit of control systems or when process designers and control designers work separately.

The contributions of this paper are: (i) a novel optimization framework for control structure selection is developed using the perfect control concept, (ii) the new optimization framework explicitly establishes a trade-off between conflicting and competing control objectives, (iii) state controllability of the process is ensured using a steady-state inversely controlled process model. In addition, the method is not limited to the linearization region, (iv) the proposed optimization framework features significant complexity reduction as the design of controllers is disentangled from the problem formulation, (v) engineering insights and heuristics are coupled with the optimization framework to ensure that the optimization results are feasible and consistent.

Use of an inverse process model decouples control structure selection from the design of the controller itself. To do this, the procedure generates an optimal structure and determines that a controller exists in principle, which is capable of delivering the optimal performance. Hence, it provides an upper bound for the behavior of the controller. It then delegates the design of the controllers to control experts to match a practical controller as closely as possible to the benchmark. Thus, while the methodology systematically addresses the problem, the formulation is practicable and scalable. 
The paper is organized as follows. Section 2 modifies the conventional optimization framework and develops a new optimization framework based on the concept of perfect control. The section explains why such an approach can contribute to the plant-wide control structure problem and how the optimization problem is formulated and solved. In Section 3, the proposed optimization framework is benchmarked using an industrial example of a distillation train. The results of the case study are presented in Sections 4. Discussion and comparison with the base case are presented in Section 5.

\section{Methodology}

Section 2.1 of the text below describes the conventional optimization framework for the selection of plant-wide control structures. The paper aims to improve and reduce the complexity of the conventional optimization framework using the notion of perfect control. The mathematical formulation of the conventional optimization framework is presented and modified in Section 2.2 in order to develop a new optimization framework. Section 2.3 discusses state controllability. Section 2.4 explains the multi-objective function. Finally, the optimized control structure must be combined with the inventory controls, as explained in Section 2.5.

\subsection{Conventional optimization framework}

The prior literature on design of control structures for chemical process involves structural decisions regarding the selection of controlled variables, manipulated variables, type of controller, and parametric decisions regarding controller parameters. These decisions have impacts on the performance of that process and a systematic framework is needed in order to generate and screen the alternative control structures. A variety of different optimization and synthesis tools has been suggested by researchers for the selection of control structures. Sakizlis et al., (2004) classified these studies based on the employed objective function which could be (i) a multi-objective steady-state objective function or (ii) a single dynamic objective function. Most of these approaches can be describe schematically by Fig. 1 . However, these methods differ in the details of modeling and the suggested objective functions.

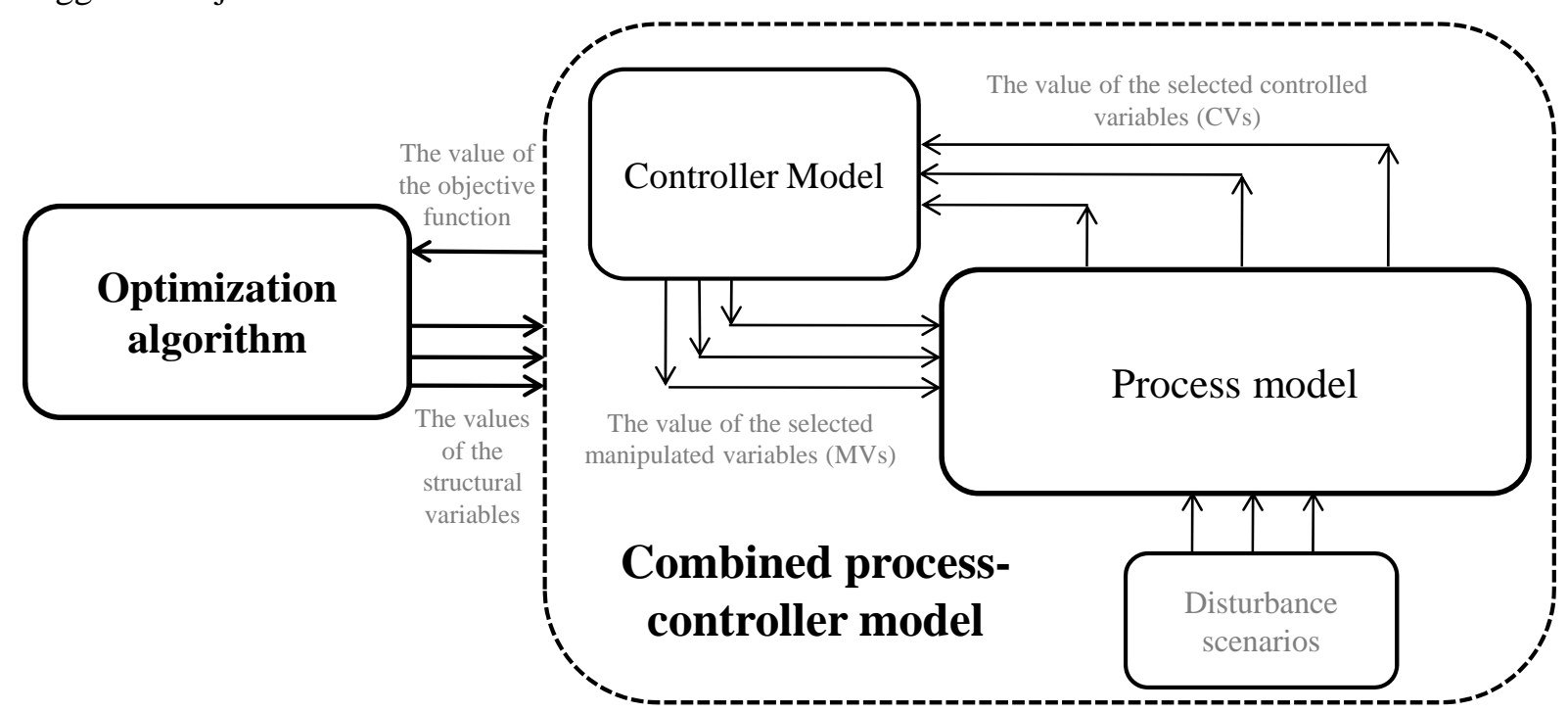

Fig. 1. The conventional optimization framework for the optimization of control structures

Ideally, the optimization framework should include a superstructure for the control system. This superstructure is shown by the dotted envelope in Fig. 1 and appears as sets of equality and inequality constraints in the optimization formulation. The superstructure enables all possible combinations of decisions for that control structure. The control design decisions include selection of controlled variables, manipulated variables, and partitioning (or in the case of single-input single-output control systems, pairing) them. In addition for each partition, a controller should be designed and its tuning parameters need to be optimized. The information flow of the conventional optimization framework (Fig. 1) is as follows: 
1. In each iteration of the optimization search, the trial values of the optimization variables are decided. These values are imported and the corresponding variables are fixed in the superstructure.

2. By fixing the optimization variables, the superstructure will be reduced into a combined model of the process and its control system.

3. Then, this design must be benchmarked against the disturbance scenarios in order to evaluate its performance. The control system detects the effects of the disturbances on the process through measuring the selected controlled variables. Then, the selected manipulated variables are adjusted in order to reject the undesirable effects of the disturbances.

4. The performance of the control system strongly depends on the above-mentioned decisions regarding the selections of the manipulated variables, the controlled variables and the controllers. This performance may be described by different criteria such as deviation of controlled variables from their setpoints, changes in the manipulation variables, or the economic losses due to disturbances. Based on these criteria, the value of the performance is calculated and reported to the optimization algorithm.

5. Based on the value of the objective function the optimization algorithm decides on improving the optimization variables or terminating the calculations.

In the conventional framework of Fig. 1, the most challenging decisions concern the design of the controllers. This is because there is no general agreement between researchers on the criteria for the selection of the controller type. Some researchers (e.g. Luyben 2004) emphasize the simplicity and robustness of the conventional multi-loop control systems and criticize the reliability and costs of modern types. On the other side of this discussion, other researchers (Rawlings 2008; Stephanopoulos 2000) argue the economic advantages of model-based control systems and their systematic approach for handling constraint violations. In addition, they criticize the economic disadvantages of the constant-setpoint policy in decentralized control systems. Furthermore, the practical difficulty is that there is no straightforward method for considering alternative controller types in the optimization formulation. Often, the type of the controllers is fixed and only the tuning parameters of those controllers are included in the optimization variables. The other difficulty is that considering controllers in the optimization framework requires a dynamic model for the process and control superstructure, which can be relatively expensive and time-consuming to develop.

In the following Sections 2.2-2.4, a new optimization framework is developed based on the inverse of the steady-state process model. The design of controllers is disentangled from the optimization framework and an upper bound of the process performance is calculated for the selected controlled and manipulated variables.

\subsection{Mathematical formulation}

In the following, Section 2.2.1 reviews the mathematical formulation of the conventional optimization framework, which uses a combined model of the process and its controllers. Section 2.2.2 modifies the conventional framework and a steady-state Inversely Controlled Process Model (ICPM) is embedded in the new optimization framework in order to reduce the problem complexity.

\subsubsection{Conventional optimization framework for integrated design and control of chemical processes}

The conventional optimization framework for selection of control structures (Fig.1) can be formulated as a stochastic mixed-integer dynamic optimization problem using a set of differential algebraic equation (DAEs) as follows (Sakizlis et al. 2004): 


$$
\min E\left\{J\left(\boldsymbol{\vartheta}, \chi_{\boldsymbol{c}}, \boldsymbol{\mu}(t)\right)\right\} \quad \text { Problem I }
$$

Subject to:

$$
\begin{gathered}
\boldsymbol{f}\left[\dot{\boldsymbol{x}}(t), \boldsymbol{x}(t), \boldsymbol{z}(t), \boldsymbol{u}(t), \boldsymbol{y}(t), \chi_{\boldsymbol{p}}, \boldsymbol{p}, \boldsymbol{z}(t)\right]=0 \\
\boldsymbol{h}\left[\boldsymbol{x}(t), \boldsymbol{z}(t), \boldsymbol{u}(t), \boldsymbol{y}(t), \chi_{\boldsymbol{p}}, \boldsymbol{p}\right]=0 \\
\boldsymbol{g}\left[\boldsymbol{x}(t), \boldsymbol{z}(t), \boldsymbol{u}(t), \boldsymbol{y}(t), \chi_{\boldsymbol{p}}, \boldsymbol{p}\right] \leq 0 \\
\boldsymbol{\theta}\left[\dot{\zeta}(t), \boldsymbol{\zeta}(t), \boldsymbol{\gamma}(t), \boldsymbol{x}(t), \boldsymbol{z}(t), \boldsymbol{y}(t), \boldsymbol{u}(t), \chi_{\boldsymbol{c}}, \boldsymbol{\vartheta}\right]=0 \\
\boldsymbol{\varphi}\left[\boldsymbol{x}(t), \boldsymbol{u}(t), \boldsymbol{y}(t), \zeta(t), \boldsymbol{\gamma}(t), \chi_{\boldsymbol{c}}, \boldsymbol{\vartheta}\right]=0 \\
\psi[\boldsymbol{\mu}(t)]=0
\end{gathered}
$$

In the above, $\boldsymbol{x}(t)$ is the vector ${ }^{1}$ of process differential variables, $\mathbf{z}(t)$ is the vector of process algebraic variables, $\boldsymbol{u}(t)$ is the vector of candidate manipulated variables, $\boldsymbol{y}(t)$ is the vector of candidate controlled variables, $\boldsymbol{p}$ is the vector of process parameters, $\zeta(t)$ is the vector of control differential variables, $\boldsymbol{\gamma}(t)$ is the vector of control algebraic variables, $\boldsymbol{\vartheta}$ is the vector of control parameters, $\boldsymbol{\mu}(t)$ is the vector of stochastic disturbance variables, $\chi_{\boldsymbol{p}}$ is the vector of structural process variables, $\chi_{c}$ is the vector of structural control variables. While $\chi_{p}$ and $\chi_{c}$ are vectors of integer variables, the rest of variables are continuous.

In addition, $\boldsymbol{f}[]=0$ is the vector of process differential equations, $\boldsymbol{h}[]=0$ is the vector of process algebraic equations, $\boldsymbol{g}[] \leq 0$ is the vector inequality constraints, $\boldsymbol{\theta}[]=0$ is the vector of control differential equations, $\varphi[]=0$ is the vector of control algebraic equations, $\Omega[]=0$ is the vector of equations for disturbances. The expected value $E\{\}$ of the objective function $J[$ ] should be minimized. Examples of objective functions are given in Section 2.4.

In a sequential design, where the process design is already decided, the decisions left for the control designer are $\chi_{\boldsymbol{c}}$ the vector of structural control variables, $\boldsymbol{\vartheta}$ the vector of controller parameters and the control law equations $\boldsymbol{\theta}$ [ ] and $\boldsymbol{\varphi}$ [] . The proposed framework simplifies the above formulation using a steady-state inversely controlled process model, discussed in the next section.

\subsubsection{Mathematical formulation of the inversely controlled process model}

In order to disentangle the design of controllers, their algebraic and differential equations $(\boldsymbol{\theta}[]=0$ and $\boldsymbol{\varphi}[]=0)$ must be replaced by perfect control equations, which ensure that the selected controlled variables are constant at their desired values:

$$
y_{i}(t)=\eta_{i}
$$

where $y_{i}(t)$ is the selected controlled variable and $\eta_{i}$ is the corresponding desired setpoint. Furthermore, using the steady-state assumption, the time dependency of the variables is ignored and the differential variables are set equal to zero in the formulation of Problem I. Therefore, Problem II consists of only algebraic equations (AEs). Since the control structure is being decided, different controlled variables may be selected during the optimization search, which represent different perfect controls. Therefore, each candidate control structure corresponds to a different set of AEs. The other issue is that for a specific set of perfect control equations some constraints should be implemented in order to ensure that a consistent set of degrees of freedom is chosen. Therefore, the modified formulation of the optimization framework can be expressed as follows:

\footnotetext{
${ }^{1}$ In this article, bold characters are reserved for vectors, and italic characters are scalar.
} 


$$
\min E\left\{J\left(\chi_{c}, \boldsymbol{\mu}\right)\right\} \quad \text { Problem II }
$$

subject to:

$$
\begin{gathered}
\boldsymbol{f}\left[\boldsymbol{x}, \boldsymbol{z}, \boldsymbol{u}, \boldsymbol{y}, \chi_{\boldsymbol{p}}, \boldsymbol{p}\right]=0 \\
\boldsymbol{h}\left[\boldsymbol{x}, \boldsymbol{z}, \boldsymbol{u}, \boldsymbol{y}, \chi_{\boldsymbol{p}}, \boldsymbol{p}\right]=0 \\
\boldsymbol{g}\left[\boldsymbol{x}, \boldsymbol{z}, \boldsymbol{u}, \boldsymbol{y}, \chi_{\boldsymbol{p}}, \boldsymbol{p}\right] \leq 0 \\
\psi[\boldsymbol{\mu}]=0 \\
\chi_{\boldsymbol{c}, \boldsymbol{k}} \times\left(y_{i}-\eta_{i}\right)=0 \\
\boldsymbol{\Omega}\left(\chi_{\boldsymbol{c}, \boldsymbol{k}}\right) \geq 0 \quad k \in K
\end{gathered}
$$

In the above, $\chi_{c, k}$ are binary variables for selection of controlled variables. The value of $\chi_{c, k}=$ 1 includes the corresponding perfect control equation in the optimization constraints. The value of $\chi_{c, k}=0$ excludes the corresponding perfect control equation from the optimization constraints. $\boldsymbol{\Omega}()$ represents vector of inequality constraints which may correspond to degrees of freedom or the requirement for a specific control strategy (e.g. composition control). The complexity reduction described above limits the optimization variables to structural variables $\chi_{c}$ only. Problem II can be solved using integer programming methods including Genetic Algorithm.

The constraints in Problem II represent a time-independent mathematical model in which the values of manipulated variables are calculated from the desired values of the controlled variables, hence the process model is inverted. The above optimization framework conforms to the results from Halvorsen et al. (2003), which state that when a disturbance scenario realizes itself, the optimal values of manipulated variables should be calculated from the desired value of controlled variables.

Fig. 2 shows the concept of the inversely controlled process model. The model of the controller has been replaced with an equation representing perfect control which enables the directions of the information flows in the optimization framework to be reversed from the controlled variables (CVs) to the manipulated variables (MVs).

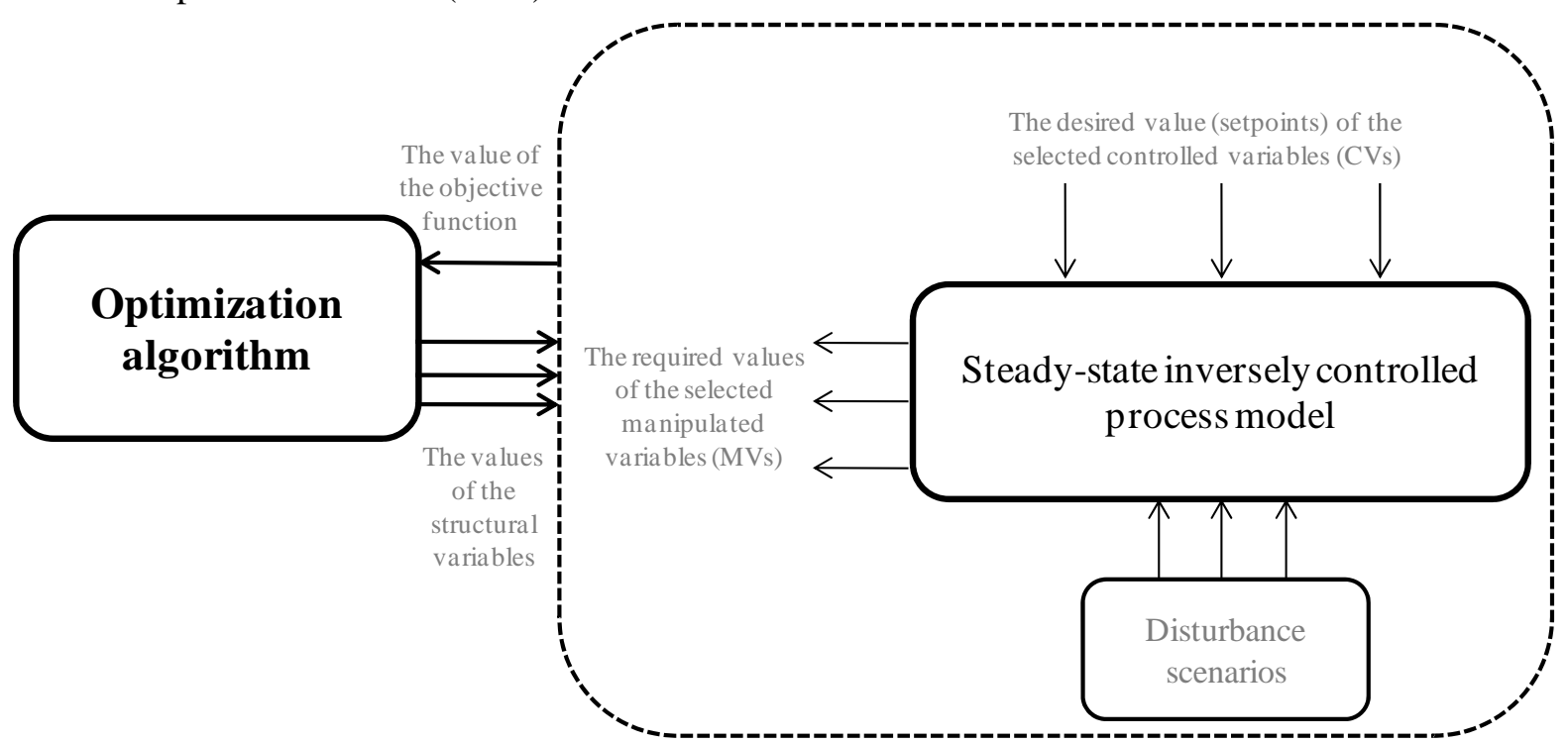

Fig. 2. The proposed optimization framework for optimal selection of control structures using an inversely controlled process model

Firstly, the optimization algorithm decides on candidate values of the structural optimization variables. These are integer variables which concern structural decisions, i.e., which variable is going to be controlled and which variable is going to be manipulated. When disturbances occur, the values of controlled variables are maintained constant using perfect control equations, while the values of the manipulated variables are adjusted in order to reject the disturbances. Then, the objective function and constraints can be evaluated and reported to the optimization algorithm. The advantage of the 
proposed framework in Problem II is significantly lower modelling effort over conventional approaches in Problem I, and therefore the new framework is more scalable to large industrial applications. Furthermore, the application of a steady-state inversely controlled process model ensures the ability to bring the system from initial steady-state to final steady state within an finite time which conforms to the notion of state controllability.(Yuan et al. 2010). In the new framework the selection of controlled variables, expected disturbance portfolio, and the manipulated variables used to reject the disturbances are the primary decisions that need to be considered.

\subsection{State controllability}

The application of a steady-state inversely controlled process model in the new optimization framework ensures the ability to bring the system from initial steady-state to final steady state within an finite time which conforms to the notion of state controllability.(Garcia 1982; Yuan et al. 2010). However, a question may arise that in the case where the inversion of the process model is not possible, the optimization will encounter problems in its calculations. Perkins and Wong (1985) summarized the scenarios in which the inversion of a process model is limited. These are right half plane zeros, model uncertainties, manipulated variables constraints and time delays. Fortunately, none of these concerns limits the application of steady-state inverse process model. Since the process model is represented as inequality and equality constraints in the optimization framework, if the inversion of the process model is not possible, these constraints are violated, which directs the optimization algorithm toward the required changes in order to make that process functionally controllable. In addition, the time delays do not appear in the steady-state analysis.

The task of detailed design and implementation of the controller is deferred to the control engineering practitioners. The structure and target closed loop performance are specified from the optimization framework. It is then for the control engineer to set up that structure and then devise a controller which most closely meets the performance from the range of control types available to him or her. The above design philosophy is consistent with the approaches developed over the last 20 years towards control loop performance assessment for already-operating control loops (Qin 1998; Jelali 2006). Typically, a control loop is benchmarked against the best achievable performance, for example using the minimum variable controller benchmark. The control practitioners are then responsible for the engineering and tuning work to meet that target performance.

\subsection{Objective functions and optimization variables}

As discussed, there are many alternative perfect control systems. Establishing criteria for selection between these alternatives is an elusive task. Edgar (2004) recognized the modern control systems as vital elements of production planning, emphasizing the fact that design of these control systems need to have a tangible relation to the production economy. Luyben (2004) discussed that improving the steady-state economy of the production reduces the dynamic performance of the process. In order to minimize the energy consumption and improve economy, the chemical processes must approach reversible conditions, in which entropy production is minimized. However, reversible processes requires negligible driving forces (such as difference in compositions or temperatures), and small driving forces lower the ability of the control system to reject disturbances. Hence, reversibility degrades the dynamic performance of the process. In summary, the utopian design, in which the process economics and dynamic performance are simultaneously at their maximum values, is infeasible and the optimal design should establish a compromise between economics and dynamic performance of the process.

The solving strategies can be classified based on the chosen objective function as steady state or dynamic, single objective or multi-objective (Sakizlis et al. 2004). In this research, a multi-objective function is considered that requires only the steady-state process model, while the state controllability of the process is ensured. The proposed objective functions are listed in Table 1. As will be discussed in the following, it is possible to extract information about performance from a steady-state model, in order to establish a tradeoff between control and economic objectives. 


\section{Table 1}

Objective functions for optimization of the control structures

$J_{1}=$ minimization of the deviations in the quality and quantity of products

$J_{2}=$ minimization of the deviations in manipulated variables

$J_{3}=$ minimization of the deviations in the state variables of process

$J_{4}=$ minimization of the economic losses due to disturbances

The implication of the first objective, $J_{1}$, is that maintaining the controlled variables at their setpoints must minimize changes in the quality and quantity of products. Under perfect control, their deviations from setpoints are zero. However, often quality and quantity are controlled inferentially by maintaining other controlled variables (typically temperature) constant. In that case, the main question is to determine which variable best represents the original controlled variable. The first objective has another implication; if the disturbance scenarios result in violation of constraints, this objective minimizes the required back-off from constraints (Perkins 1996).

The second objective, $J_{2}$, aims to minimize changes in manipulated variables in response to disturbances or setpoint changes. The suppression of changes in the manipulated variables is desirable to avoid valve saturation (thus, maintaining controllability), to minimize the consumption of resources associated with manipulated variables (e.g. utilities, feedstock, or solvents), to reduce the interaction between controllers, and to minimize the required time for disturbance rejection (Qin and Badgwell 2003; McAvoy 1999).

The third objective, $J_{3}$, measures the response of the state variables in a perfectly controlled process to disturbances when the controlled variables are maintained at their setpoints. An example of this objective is the change in the temperature profile of a distillation column when flow or composition of feed is disturbed.

The fourth objective, $J_{4}$, concerns steady-state economic losses, i.e. the decrease in the profitability due to disturbances. The origin of this objective refers to the notion of self-optimizing control and its implication is that maintaining the optimal controlled variables at their setpoints must minimize economic losses in the presence of disturbances, (Skogestad 2000).

The difficulty associated with multi-objective optimization of control structure selection is that the different economic or performance objectives are incommensurable, i.e. it is difficult to aggregate their value as a single objective value. In this research, a goal objective function is programmed (Jones 2010). In goal programming, each objective function is given a goal or target value. The deviation from these target values are then minimized as follows:

$$
\min \left(\max \left[J_{w}-\operatorname{Target}_{w}\right]\right) \quad w=1 \ldots 4
$$

Selection of target values for the objective functions of Table 1. is straight forward, because these targets have ideally the value of zero:

$$
\text { Target }_{w}=0 \quad w=1 \ldots 4
$$

In each iteration of the optimization search, the min-max operator minimizes the objective function which has the largest deviation from its target value. Substitution of equation (2) in Problem II results in the following problem formulation:

$$
\min \left(\max \left[J_{w}\right]\right) \quad \text { Problem III }
$$

subject to:

$$
\begin{aligned}
& J_{w}\left(\chi_{\boldsymbol{c}}\right)=E\left\{J^{\prime}{ }_{w}\left(\chi_{\boldsymbol{c}}, \boldsymbol{\mu}\right)\right\} \quad w=1 \ldots 4 \\
& \boldsymbol{f}\left[\boldsymbol{x}, \boldsymbol{z}, \boldsymbol{u}, \boldsymbol{y}, \chi_{\boldsymbol{p}}, \boldsymbol{p}\right]=0 \\
& \boldsymbol{h}\left[\boldsymbol{x}, \boldsymbol{z}, \boldsymbol{u}, \boldsymbol{y}, \boldsymbol{\chi}_{\boldsymbol{p}}, \boldsymbol{p}\right]=0 \\
& \boldsymbol{g}\left[\boldsymbol{x}, \boldsymbol{z}, \boldsymbol{u}, \boldsymbol{y}, \chi_{\boldsymbol{p}}, \boldsymbol{p}\right] \leq 0
\end{aligned}
$$




$$
\begin{gathered}
\psi[\boldsymbol{\mu}]=0 \\
\chi_{\boldsymbol{c}, \boldsymbol{k}} \times\left(y_{i}-\eta_{i}\right)=0 \\
\Omega\left(\chi_{\boldsymbol{c}, \boldsymbol{k}}\right) \geq 0 \quad k \in K
\end{gathered}
$$

In problem III, $J_{w}(w=1, \ldots, 4)$ are the expected values of corresponding objective values $J^{\prime}{ }_{w}$ for different disturbance scenarios, and is constructed by adding these objective values weighted by the likelihood of each disturbance scenario, (Sahinidis 2004). The objectives of Problem III, $J_{w}\left(\chi_{c}\right)$, has only integer variables in their arguments. All the continuous variables are implicit and included in the optimization constraints and can be handled using an algebraic equation solver as will be discussed in section 3.3.1.The reason is that the problem of control structure selection concerns about the effects of disturbances on the same process when different sets of controlled and manipulated variables are selected.

\subsection{Engineering insights and heuristics: dynamic degrees of freedom and design of inventory control systems}

Luyben (1999) presented a review of the heuristics and engineering insights for inventory controls, developed during decades of engineering practice. In summary, these rules suggest firstly designing the throughput manipulator for the plant and then the inventory control systems radiate from this point, i.e., are in the opposite direction of the flow in the upstream and in the direction of the flow in the downstream of the throughput manipulation point (Georgakis and Price 1993; Georgakis et al. 1994). Downs (1992) emphasized that the inventory control analysis must be considered for each chemical component throughout the process. Luyben (1994) recommended that a flow in the recycle stream must be fixed in order to eliminate the possibility of the snowball effect, i.e. coordinated disturbances to the mass or energy flows around the recycle.

Since liquid levels and gas inventories do not appear in a steady-state model, these variables must be included separately in the control structure. The application of an inverse steady-state process model decomposes the problem of control structure selection into two smaller sub-problems. One subproblem is addressing the task of designing inventory controls, and the other sub-problem systematically addresses the steady-state optimizing control system. A question may arise about whether these sub-problems can be addressed independently. The answer is to some extent negative. Since the available manipulated variables are shared between steady-state controlled variables and inventory controlled variables, the set of candidate steady-state controlled variables must be arranged in such a way that, if any of the candidate controlled variables are selected by the optimization algorithm, the required manipulated variables are available and none of inventory variables will be left uncontrolled. Otherwise, the infeasible controlled variable must be removed from the set of candidate controlled variables and the optimization program should be run again.

\section{Case study: optimal control structure selection for a distillation trains}

The proposed optimization framework is applied to the case of an industrial distillation train in an olefin plant. The aim of this case study is to illustrate the implementation of framework and to demonstrate its performance. The process description is presented in Section 3.1. The software tools used in implementation of the proposed optimization framework are presented in Section 3.2. The optimization variables and constraints corresponding to degree of freedom analysis and the requirement for composition control are presented in Section 3.3. The case study is adapted from the olefin plant of Arak Petrochemical Co., Arak, Iran.

\subsection{Process description of pyrolysis gasoline hydrogenation (PGH) plant}

The process description for the overall olefin plant is available in the literature (Kirk-Othmer 2007). A section of this process concerns the treatment of pyrolysis gasoline from which the case study of this paper is selected. This section is called pyrolysis gasoline hydrogenation (PGH) section and is shown in Fig. 3. 
In the olefin processes, the products of the cracking reactions of the liquid feedstock include a blend with properties very similar to gasoline. The disadvantage of this product is that the dissolved light olefins are highly reactive with risk of polymerization stored untreated. Therefore, this blend must be saturated by hydrogenation reactions. The reaction conditions are $24 \mathrm{bar}$ and $140^{\circ} \mathrm{C}$. The incondensable components that mostly consist of hydrogen are separated in a series of two separator drums which are operated in hot and cold conditions. The overhead vapors of the first separator are cooled using an air-cooler and a cooling water heat exchanger in order to minimize the hydrocarbon loss in the fuel gas stream. Then, the condensates from the bottom of these two separator drums will be resolved in a distillation train into $C_{5}, C_{6}, C_{7}^{+}$and heavy-ends products. The distillation train is studied in this paper and its schematic is shown by the dotted envelope on the right hand side of Fig. 3.

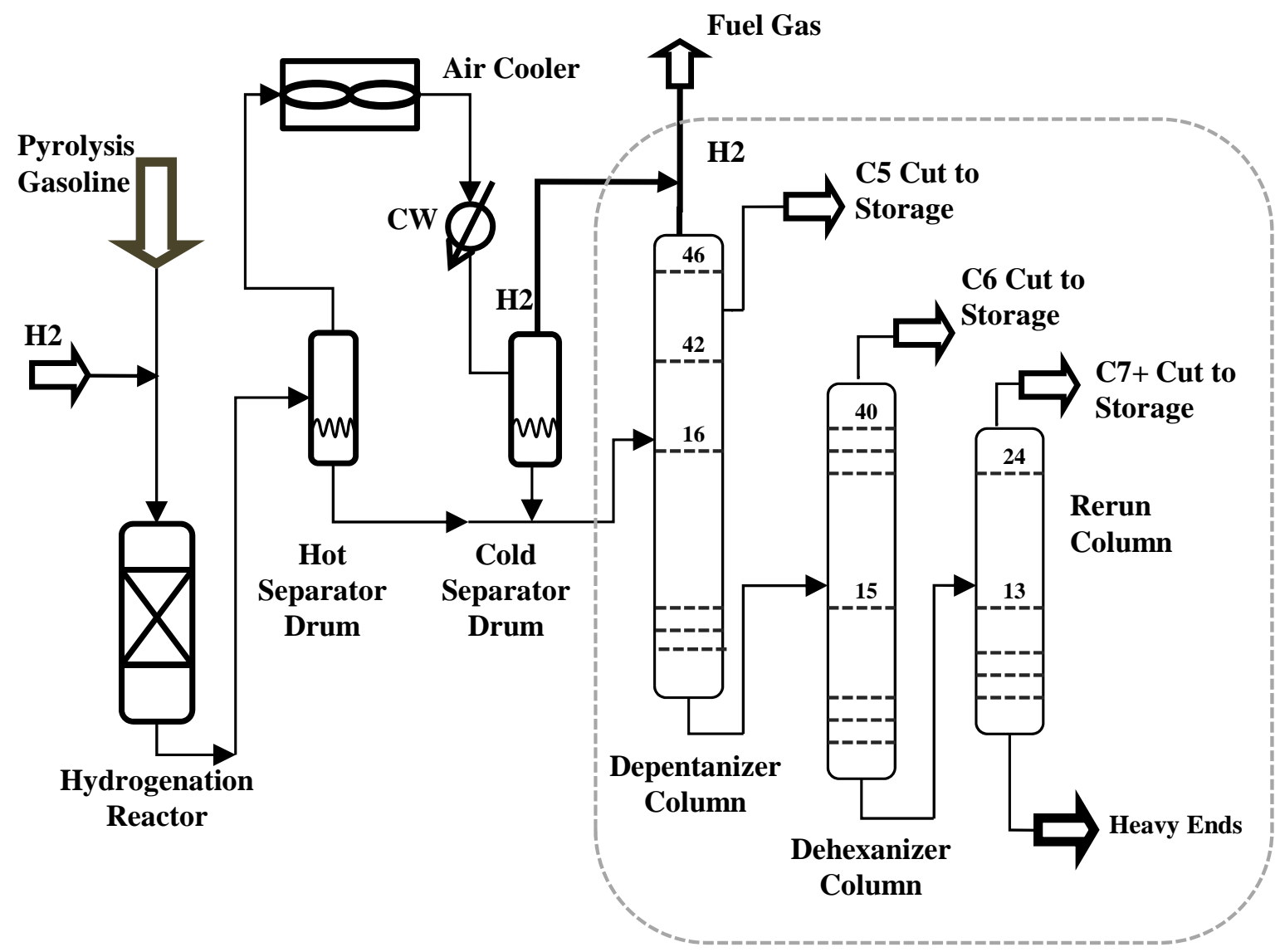

Fig. 3. PGH plant; the framed part of the flowsheet is selected for the case study.

The first distillation column is the depentanizer column. This column has a partial reflux configuration and the gaseous overhead product is mostly hydrogen. The main product is the $C_{5}$ cut, and is withdrawn as the side stream. The bottom stream is fed to the dehexanizer column. The $C_{6}$ cut is produced in the overhead of the dehexanizer column and the bottom stream is fed to the rerun column which is operated under vacuum conditions. This column resolves its feed to $C_{7}^{+}$and heavyends streams.

\subsection{Optimization variables and constraints}

As discussed in Section 2.2.2. the new optimization framework (Fig. 2), limits the optimization variables to structural variables $\chi_{\boldsymbol{c}}$. In the following, the inventory design methods described in Section 2.6 are applied to the case study and technical consideration of composition control is spelled out. 
Table 2.

List of binary optimization variables for the PGH case study.

$\chi_{c j T t}$ : a binary variable which represents selection of the temperature

of a column tray as a controlled variable, and corresponds to a specific perfect control equation in Problem II

\begin{tabular}{cl}
\hline $\begin{array}{c}\text { Depentanizer: } \\
\text { C5 }\end{array}$ & $\left\{\chi_{c 5, T 1} \ldots \chi_{c 5, T 46}\right\}$ \\
Dehexanizer: & \\
C6 & $\left\{\chi_{c 6, T 1} \ldots \chi_{c 6, T 40}\right\}$ \\
Rerun: & $\left\{\chi_{c 7, T 1} \ldots \chi_{c 7, T 24}\right\}$ \\
C7 &
\end{tabular}

$\chi_{\boldsymbol{c j}, \boldsymbol{F l o w}}$ : a binary variable which represents selection of a flow, a flowratio or a heat duty as a controlled variable, and corresponds to a specific perfect control equation in Problem II

$$
\begin{gathered}
\chi_{c 5, D}, \chi_{c 5, B}, \chi_{c 5, S}, \chi_{c 5, R}, \chi_{c 5, Q_{H}} \\
\chi_{c 5, D / F}, \chi_{c 5, B / F}, \chi_{c 5, S / F}, \chi_{c 5, R / F}, \chi_{c 5, R / D} \\
\chi_{c 6, D}, \chi_{c 6, B}, \chi_{c 6, R}, \chi_{c 6, Q_{H}} \\
\chi_{c 6, D / F}, \chi_{c 6, B / F}, \chi_{c 6, R / F}, \chi_{c 6, R / D} \\
\chi_{c 7, D}, \chi_{c 7, B}, \chi_{c 7, R}, \chi_{c 7, Q_{H}} \\
\chi_{c 7, D / F}, \chi_{c 7, B / F}, \chi_{c 7, R / F}, \chi_{c 7, R / D}
\end{gathered}
$$

Table 2 lists the optimization variables. In the steady-state inversely controlled process model, these are the candidate variables to be selected as controlled variables and maintained constant. The rational choice of these candidate variables is based on the available measurements. The index $j=5,6,7$ represents depentanizer, dehexanizer and rerun columns respectively. The notation $\chi_{\boldsymbol{c j}, \boldsymbol{T t}}$ refers to the temperature of tray number $t$ in the $j^{\text {th }}$ column. The notation $\chi_{c j, F l o w}$ refers to a flowrate in column $j$. The notations $R, D, B, S$ represent reflux, distillate, bottom, and side streams respectively. $Q_{H}$ refers to reboiler heat duty. These notations also hold for flow ratios, for example $\chi_{c 6, B / F}$ represents the ratio of bottom flowrate to feed flowrate in dehexanizer column.

\subsubsection{Design of inventory control systems}

Throughput manipulation is assumed to be present upstream and the inventory in the distillation train is controlled in the direction of flow (Georgakis and Price 1993; Georgakis et al. 1994). The implication of this assumption is that the feed of the distillation train is assumed as the disturbance source and varies independently.

The second (dehexanizer) and third (rerun) distillation columns are total reflux columns and there are five potential manipulated variables; these are the reboiler heating duty, the condenser cooling duty, the reflux flow, and the flow rates of the overhead and bottom products. However, controlling the overhead and bottom level of liquid inventories, and column pressure which represents the vapor inventory consume three manipulated variables, and two manipulated variable remain to be included in the optimization variables.

The first distillation column uses a partial condenser due to the presence of the incondensable components. The flow of the overhead vapor is used for controlling the column pressure and the condenser duty is used for the overhead liquid inventory. The side-product stream has an extra degree of freedom because the side-draw flow rate is a potential manipulated variable. This degree of freedom in addition to partial reflux flowrate and bottom flowrate provide three degrees of freedom.

\subsubsection{Composition control and inferential temperature control}

With liquid level and pressure control loops closed, the distillation column is still unstable due to composition drift (Hori and Skogestad 2007; Skogestad 2007). Ideally, composition should be measured directly. However, composition measurements can be expensive and slow. An alternative might be to specify the set point of a temperature controller (Luyben 2006). The idea behind is that changes in the selected temperature must represent the changes in the column product composition and maintaining the temperature at its setpoint will ensure that product specifications are met. The instability issue makes the composition or inferential temperature loop a top priority in a distillation column. However, if any further degree of freedom is available, it can be assigned to control a second temperature. Therefore, a set of constraints must ensure selection of at least a temperature as a controlled variable in each column. The corresponding constraints for the above discussion about inventory and composition control can be represented by following constraints: 


$$
\begin{aligned}
& \chi_{c 5, T 1}+\cdots+\chi_{c 5, T 46}+\chi_{c 5, D}+\chi_{c 5, B}+\chi_{c 5, S}+\chi_{c 5, R}+\chi_{c 5, D / F}+\chi_{c 5, B / F}+\chi_{c 5, S / F}+ \\
& +\chi_{c 5, R / F}+\chi_{c 5, R / D}+\chi_{c 5, Q_{H}}=3 \\
& \chi_{c 6, T 1}+\cdots+\chi_{c 5, T 39}+\chi_{c 6, D}+\chi_{c 6, B}+\chi_{c 6, R}+\chi_{c 6, D / F}+\chi_{c 6, B / F}+\chi_{c 6, R / F}+\chi_{c 6, R / D}+ \\
& +\chi_{c 6, Q_{H}}=2 \\
& \chi_{c 7, T 1}+\cdots+\chi_{c 5, T 24} \chi_{c 7, D}+\chi_{c 7, B}+\chi_{c 7, R}+\chi_{c 7, D / F}+\chi_{c 7, B / F}+\chi_{c 7, R / F}+\chi_{c 7, R / D}+ \\
& +\chi_{c 7, Q_{H}}=2 \\
& \chi_{c 5, T 1}+\chi_{c 5, T 2}+\cdots+\chi_{c 5, T 45}+\chi_{c 5, T 46} \geq 1 \\
& \chi_{c 6, T 1}+\chi_{c 6, T 2}+\cdots+\chi_{c 5, T 39}+\chi_{c 5, T 40} \geq 1 \\
& \chi_{c 7, T 1}+\chi_{c 7, T 2}+\cdots+\chi_{c 5, T 23}+\chi_{c 5, T 24} \geq 1
\end{aligned}
$$

Constraint (4a-c) ensures that a correct degree of freedom is selected for each distillation column. Constraint (5a-c) ensures that at least a temperature controlled variable (representing the composition control) is selected in each distillation column.

\subsection{Implementation tools and considerations}

\subsubsection{Simulation-optimization programming technique}

The simulation-optimization programming technique used in this research conformed to the optimization with implicit constraints, which has been proved efficient in process optimization using simulators (Sharifzadeh and Thornhill 2011; Caballero et al 2007; Odjo et al 2011). The process simulator has an input-output black-box relationship to the optimizer. The optimization is performed in the outer loop and the simulation is solved in the inner loop. The advantage of this method is that the number of optimization variables is limited to the degree of freedom of the simulation, (i.e., the number of independent variables which should be specified independently to run the simulator). For fixed values of the optimization variables, the equation solver of the simulator is able to calculate the remaining variables. By the convergence of the simulator's equation solver, the value of the objective function is evaluated. The disadvantage of this method is that evaluation of the objective function is computationally expensive because for each evaluation, the equation solver needs to converge.

\subsubsection{Process modeling and disturbance scenarios}

In this research, the simulation was performed using Aspen-HYSYS ${ }^{\circledR}$ and the optimization algorithm was the Genetic Algorithm $\left(\mathrm{GA}^{\circledR}\right)$ toolbox of MATLAB ${ }^{\circledR}$. The two software tools where integrated using $\mathrm{COM}^{\circledR}$ automation interface. The mathematical modeling is performed using the distillation blocks of the Aspen-HYSYS simulator. The underlying equations can be found in the documentation of the software (Aspen-HYSYS document 2009a). The pyrolysis gasoline was estimated by 34 real components, and its estimated properties were in a good agreement with the data from the plant. The modified Peng-Robinson equation of state was employed for thermodynamic calculations (AspenHYSYS document 2009b). The accuracy of the results depends on the applied modelling and optimization methods.The developed model featured a high degree of rigorousness because built-in distillation blocks and a high fidelity property package from the simulator library were used for the modeling. Furthermore, Genetic Algorithm is a stochastic global optimization method based on evolution of a population of optimization solutions and is less likely to become entrapped in local optimums, (Edgar 2001; Mitchell 1998).

The feed stream to the depentanizer column is assumed a disturbance. The feed can be represented as the mixture of four cuts of hydrocarbons: $C_{5}, C_{6}, C_{7}^{+}$and heavy-ends. In each disturbance scenario, the amount of each of these cuts in the feed stream is changed by $\pm 5 \%$. The combination of these, changes results in sixteen disturbance scenarios, which represent the operational conditions thoroughly. These disturbance scenarios are equally likely. 


\subsubsection{Constructing a steady-state inversely controlled process model in the simulator}

The distillation block in Aspen-HYSYS provides the option for defining "column specifications." These are the specifications that the equation solver tries to meet during the simulation, and are in fact the degrees of freedom of the simulation. For each specification, a desired value was set according to the operating conditions of the base-case process. Activating or deactivating these specifications provides the opportunity to add or remove perfect equations (1) and to construct the corresponding steady-state inversely controlled process model.

\subsubsection{Simulation-optimization information flow}

Fig. 4 shows the information flow of the simulation-optimization. The left-hand side block and the right-hand side block are the GA toolbox and Aspen-HYSYS simulator, respectively. The middle block is an m.file coded in MATLAB, which integrates the two software tools. In each optimization iteration, GA decides on the value of the optimization variables (Table 2). By satisfaction of constraints (4a-c), (5a-c), only seven optimization variables are equal to one and the rest are zero. The integrating code receives the value of the optimization variables, activates the corresponding column specifications, and constructs the steady-state inversely controlled process model as described in Section 3.3.3. Then, the current choice of the optimization variables must be benchmarked against disturbance scenarios. The integrating code imposes the disturbances to the inversely controlled process model by changing the feed flowrate and composition as described in Section 3.3.2. For each disturbance scenario, the corresponding values of the objective functions (Table 1) are evaluated. The value of the multi-objective function (equation 2) is constructed as explained in section 2.4 and is reported back to the GA algorithm. The GA algorithm evaluates the termination criteria and decides on improving optimization variables.

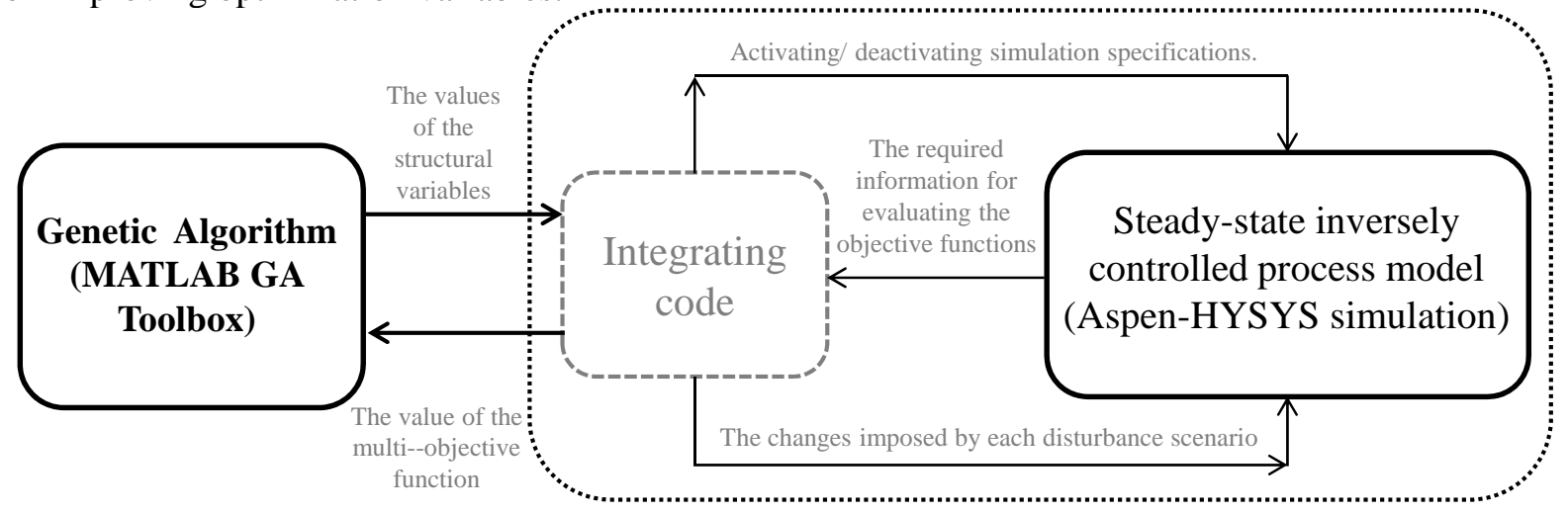

Fig. 4. Information flow of the simulation-optimization programming.

\section{Results of the case study}

\subsection{Optimized control structure}

The results of the optimization of the proposed case study of PGH distillation train are presented in this section. The implications of the optimization results and their implementation in the optimized control structure are discussed as follows.

Table 3.

The optimal controlled variables selected for the three distillation columns by the optimization framework

\begin{tabular}{|c|c|c|c|}
\hline Depentanizer Column & Dehexanizer Column & Rerun column & $\begin{array}{l}\text { Type of the } \\
\text { controlled } \\
\text { variable }\end{array}$ \\
\hline Column pressure & Column pressure & Column pressure & Inventory \\
\hline Overhead liquid level & Overhead liquid level & Overhead liquid level & Inventory \\
\hline Bottom liquid level & Bottom liquid level & Bottom liquid level & Inventory \\
\hline Temperature of the $45^{\text {th }}$ tray & Temperature of the $24^{\text {th }}$ tray & Temperature of the $6^{\text {th }}$ tray & Steady-state \\
\hline Temperature of the $33^{\text {rd }}$ tray & Reflux/Feed flow ratio & Reflux flow rate & Steady-state \\
\hline Temperature of the $10^{\text {th }}$ tray & none & none & Steady-state \\
\hline
\end{tabular}


Table 3. presents the results. The controlled variables associated with inventory designs are included in the top three rows. The bottom three rows report the controlled variables which are selected by the optimization framework. It is noteworthy that in the third column the optimizer chose to fix a degree of freedom, i.e. reflux flowrate. Therefore, the flowrate of reflux in the third (rerun) column is constant and this degree of freedom will not be available for the controller design.

In Fig. 5, the available manipulated variables are shown using control valve symbols. The controlled variables selected by the optimization algorithm are shown using dotted circles. The liquid (level) and vapor (pressure) inventory controlled variables are shown using the solid squares. These are the results of the optimization framework in conjunction with heuristics for the design of the inventory control system. These results could be directly used in a multivariable control design. However, if a multi-loop control system is being designed an appropriate pairing method such as RGA and its variants or process knowledge and insights can be employed in order to develop the overall control structure.

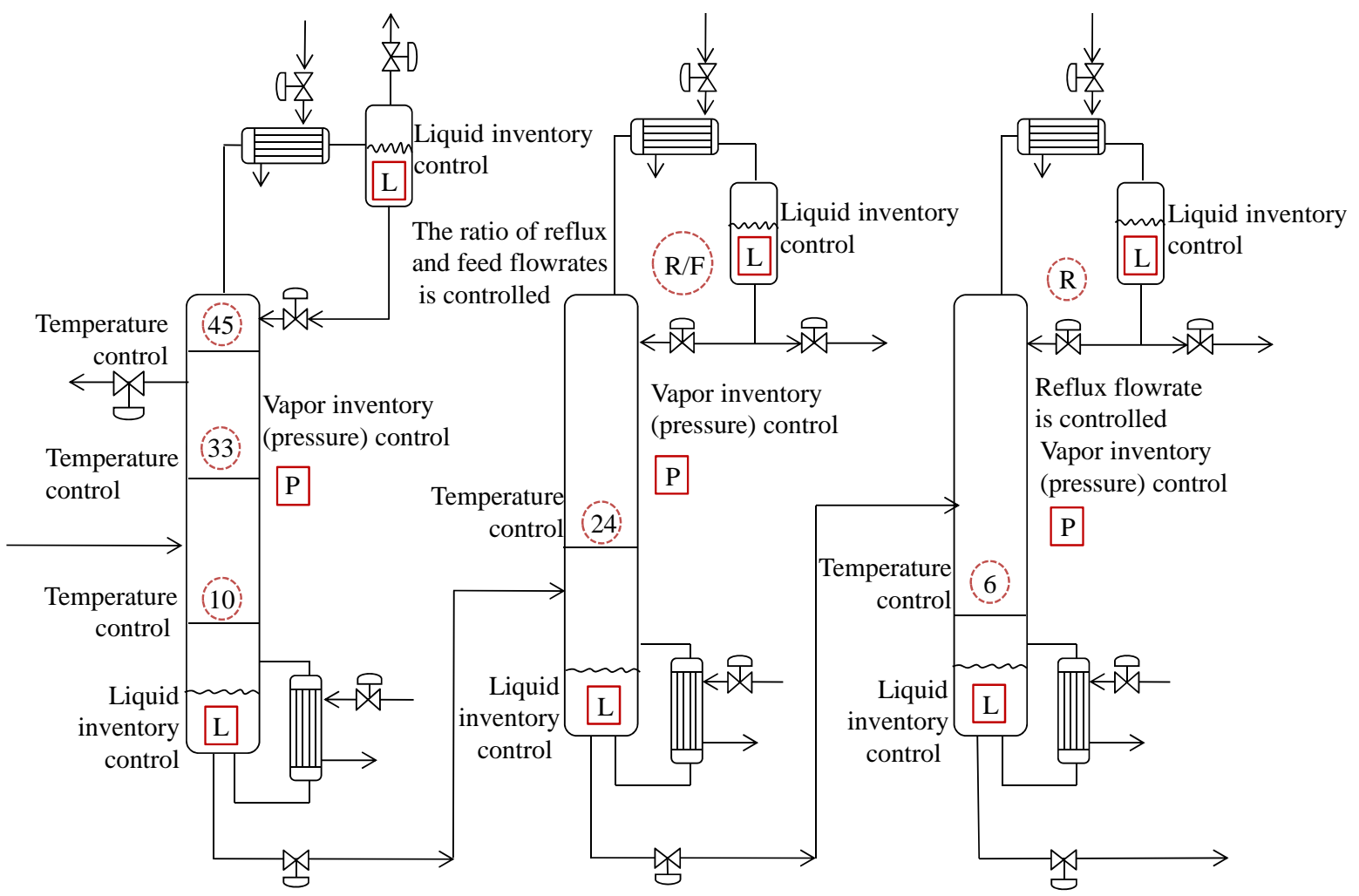

Fig. 5. The selected controlled variables using the optimization framework (dotted circles), and the inventory controlled variables (solid circles)

As discussed earlier, the detailed control system design is delegated to the process control engineers. An example of a possible multi-loop control structure for the selected controlled variables is shown in Fig. 6. Here, the controlled variables and the available manipulated variables are paired using the process insights described by Skogestad (2007) and Luyben (2006).

In the first distillation column, which has a partial condenser, the overhead vapor is used for controlling the column pressure. In the second and third distillation columns, which have total condensers, the condenser duty is paired with column pressures. These are common engineering practices for partial and total reflux columns. All of the liquid inventories are controlled by the corresponding outgoing liquid streams except the liquid inventory of the first column, in which the level of the overhead liquid inventory is controlled using the condenser duty. In addition, the design of flow controllers does not require pairing. The temperature controllers are paired with the remaining manipulated variables. 

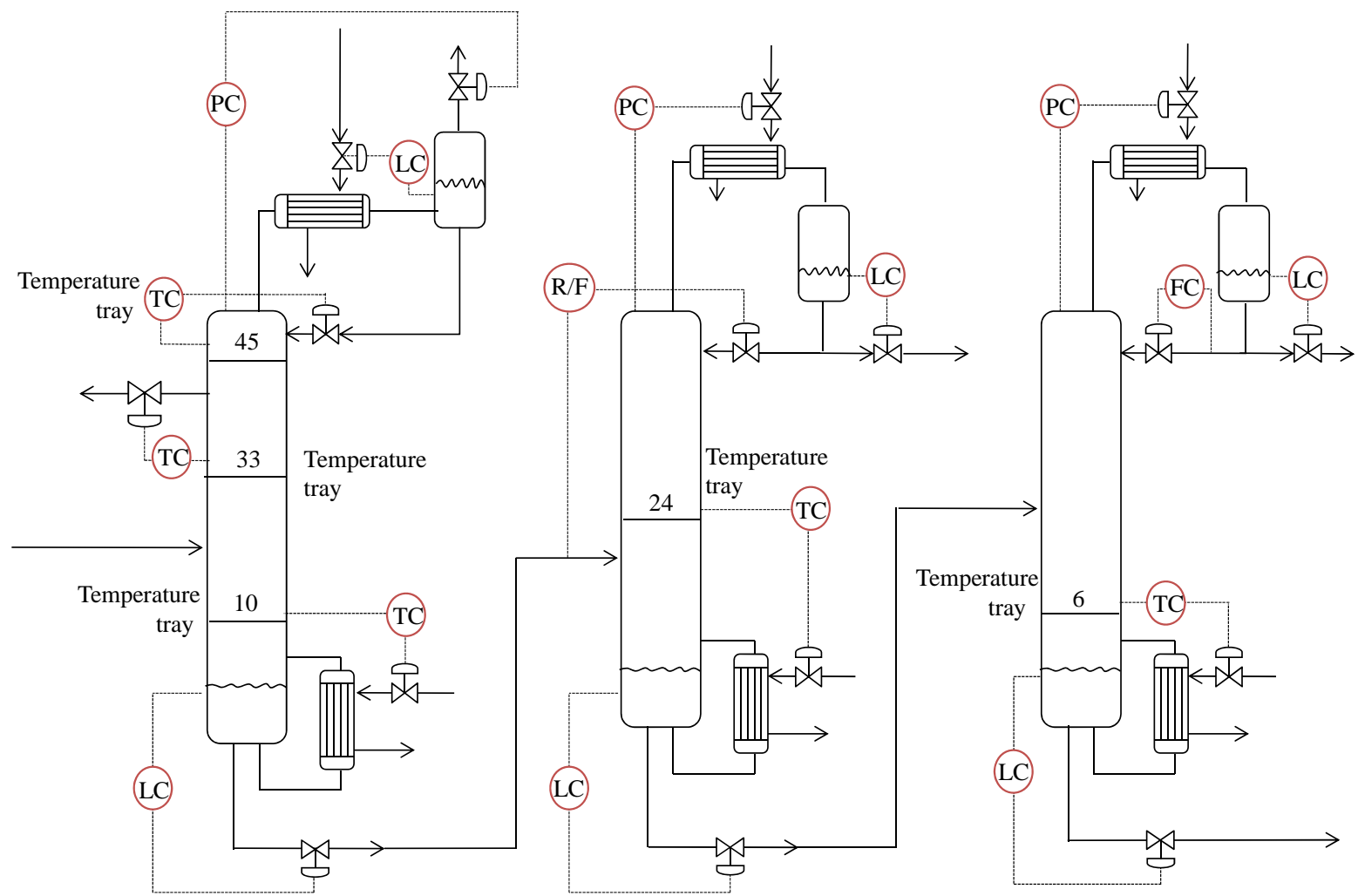

Fig. 6. The control structure for the distillation train of the PGH Process (Tray-numbering is bottomup)

\subsection{Performance comparisons}

The sixteen disturbance scenarios included a wide range of changes in the flow and the composition of the feed. Table 4 . shows the average values of the objective functions for the optimized structure and compared with the base-case unoptimized structure that will be described in Section 5. The target of the optimization is that the deviations should be zero when the disturbances are applied. The table shows an improvement over the base-case and demonstrates the trade-off between different competing objective functions, which is established by the optimization algorithm. Although the objective functions of Table 1 are competing and conflicting objectives, the optimal solution exhibits desirable properties. While the manipulated variables (Third column) are preserved from excessive movement in different disturbance scenarios, the profit losses are minimized (second column). The product specifications are met (fifth and sixth columns) and a minor change in average temperature profiles indicates short trajectories between different process steady states (fourth column).

Table 4.

The average of optimal values of the objective functions in different disturbance scenarios

\begin{tabular}{llllll}
\hline & $\begin{array}{l}\text { Average } \\
\text { changes in } \\
\text { net profit } \\
{[\%]}\end{array}$ & $\begin{array}{l}\text { Average } \\
\text { changes in } \\
\text { manipulated } \\
\text { variables [\%] }\end{array}$ & $\begin{array}{l}\text { Average } \\
\text { changes in the } \\
\text { tempreature of } \\
\text { trays [oC] }\end{array}$ & $\begin{array}{l}\text { Average changes } \\
\text { in product } \\
\text { molecular weight } \\
{[\%]}\end{array}$ & $\begin{array}{l}\text { Average } \\
\text { changes in } \\
\text { product } \\
\text { density [\%] }\end{array}$ \\
\hline $\begin{array}{l}\text { Optimized control } \\
\text { structure }\end{array}$ & -0.041 & 1.47 & 0.18 & 0.81 & 0.28 \\
\hline $\begin{array}{l}\text { Base-case control } \\
\text { structure (Section 5) }\end{array}$ & -0.223 & 0.72 & 1.17 & 9.706 & 0.837 \\
\hline
\end{tabular}

Fig. 7.a. shows the temperature profiles of the first distillation column for the 16 disturbance scenarios. Since three temperature controllers are used in the first distillation column, the temperature profiles are very similar in this column. Temperature profiles in the second and third distillation columns are shown in Figs. 7b-c, which demonstrate a satisfactory control of temperature (and inferentially composition) over the range of the 16 disturbances. 


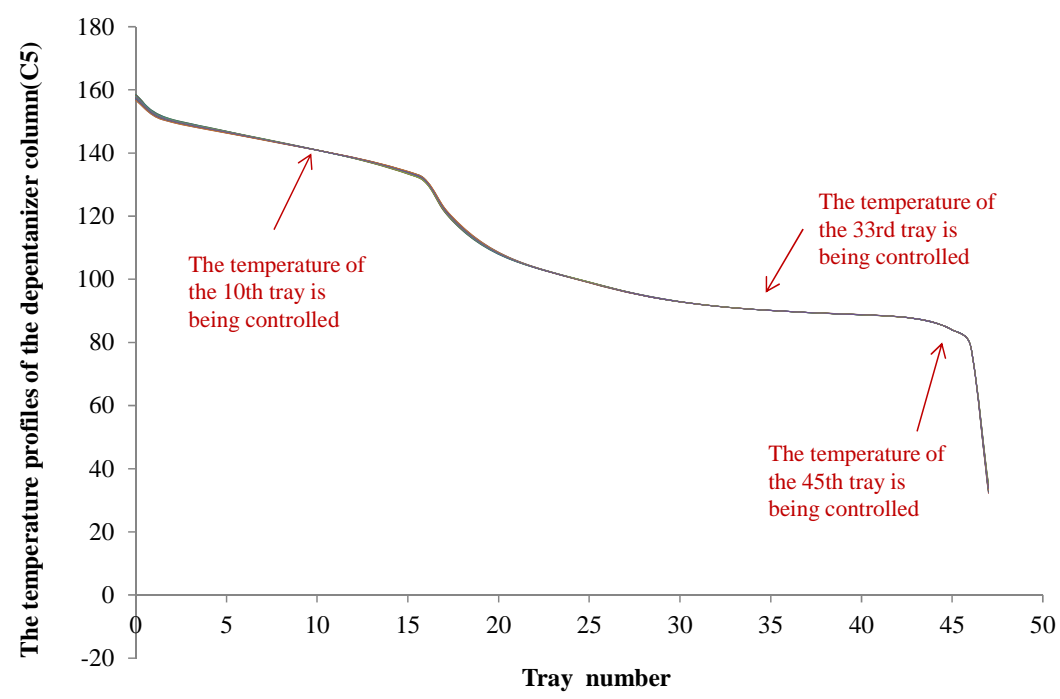

Fig. 7.a. The temperature profiles of the depentanizer column for the sixteen disturbance scenarios for the optimized control structure

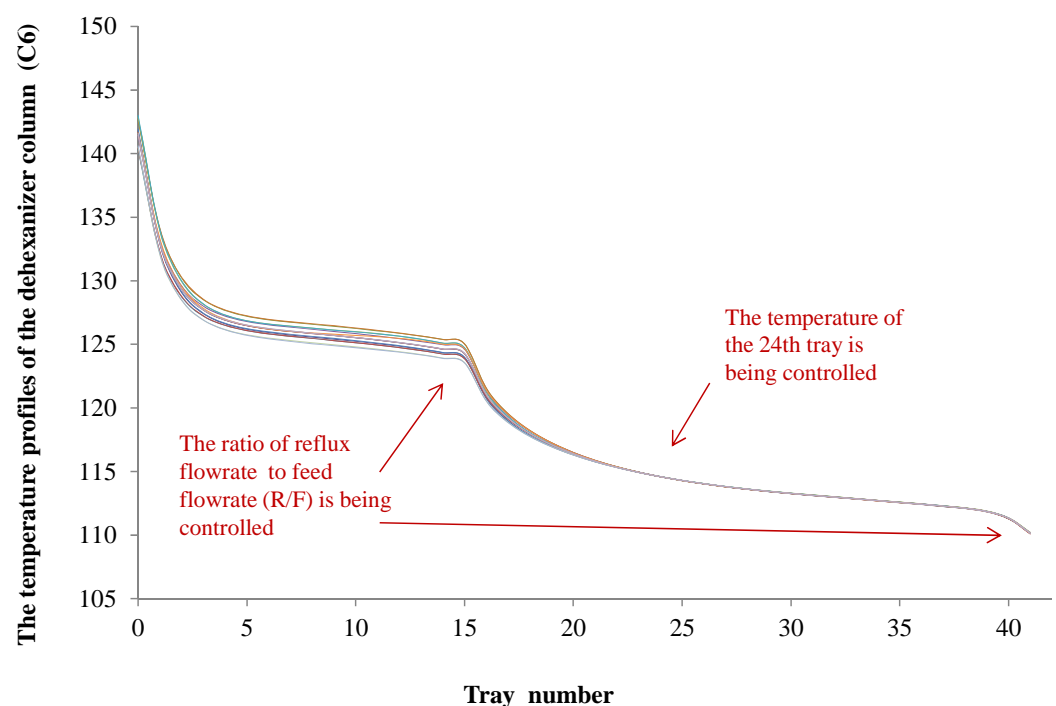

Fig. 7.b. The temperature profiles of the dehexanizer column for the sixteen disturbance scenarios for the optimized control structure

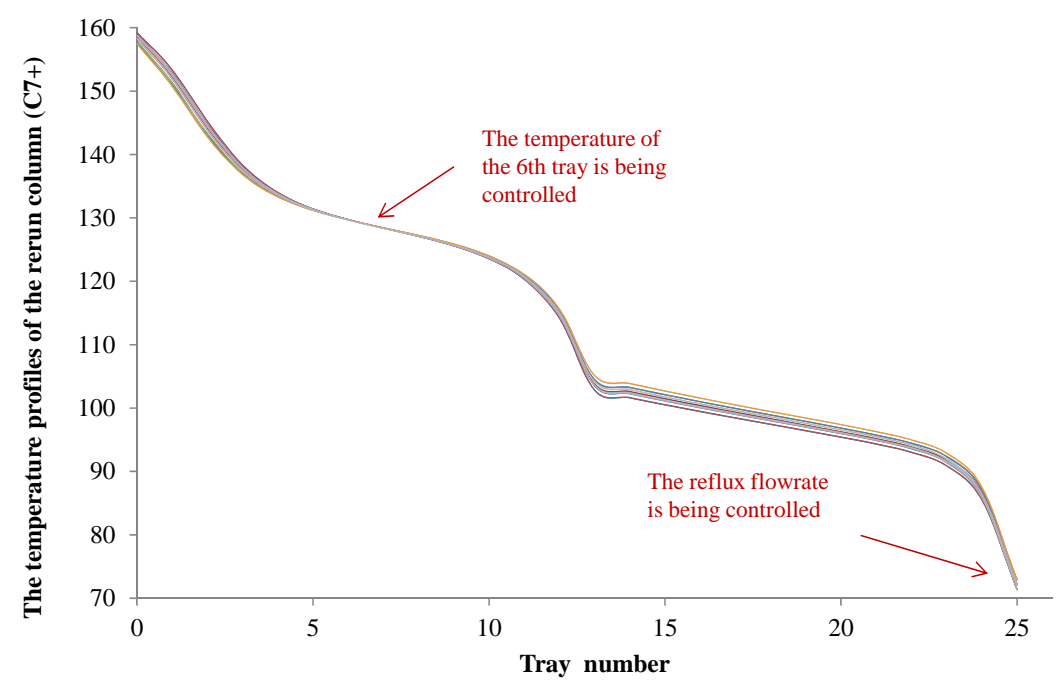

Fig. 7.c. The temperature profiles of the rerun column for the sixteen disturbance scenarios for the optimized control structure 


\section{Comparison and discussion}

In this section, the effects of the same disturbances scenarios as described in Section 3.1. are presented for a base-case (unoptimized) process. The base-case control structure is shown in Fig. 8. Key differences are in the first column, depentanizer, in which the heat duty of condenser, temperature of the side stream and the temperature of the reboiler are being controlled in the basecase design. By contrast, three inside temperatures corresponding to columns trays are being controlled in the optimized control structure. This strategy results in significant improvements of the performance of the optimized control structure, which can be investigated by comparing Fig. 7a. and Fig. 9a. The main advantage of the optimized control structure is that it minimizes the loss of hydrocarbon product with the overhead fuel-gas purge stream in the first column. In addition, for the proposed disturbance scenarios, the optimized control structure remains operable while the base-case control structure would lose its control action in some certain disturbance scenario, as shown by Fig. 9.a. the base-case design requires such a low temperatures that is not achievable using cooling water. This would show itself as saturation control valve and loss of valuable product from overhead stream.

The difference between the two structures is less pronounced in the second and third columns. The optimized control structure of the second column uses reflux/feed ratio compared to reflux flowrate in the base-case control structure. In addition, the temperature of a tray is controlled in the optimized control structure in comparison to the reboiler temperature in the base case design. The control structure of the last column only differs in the number of temperature tray.

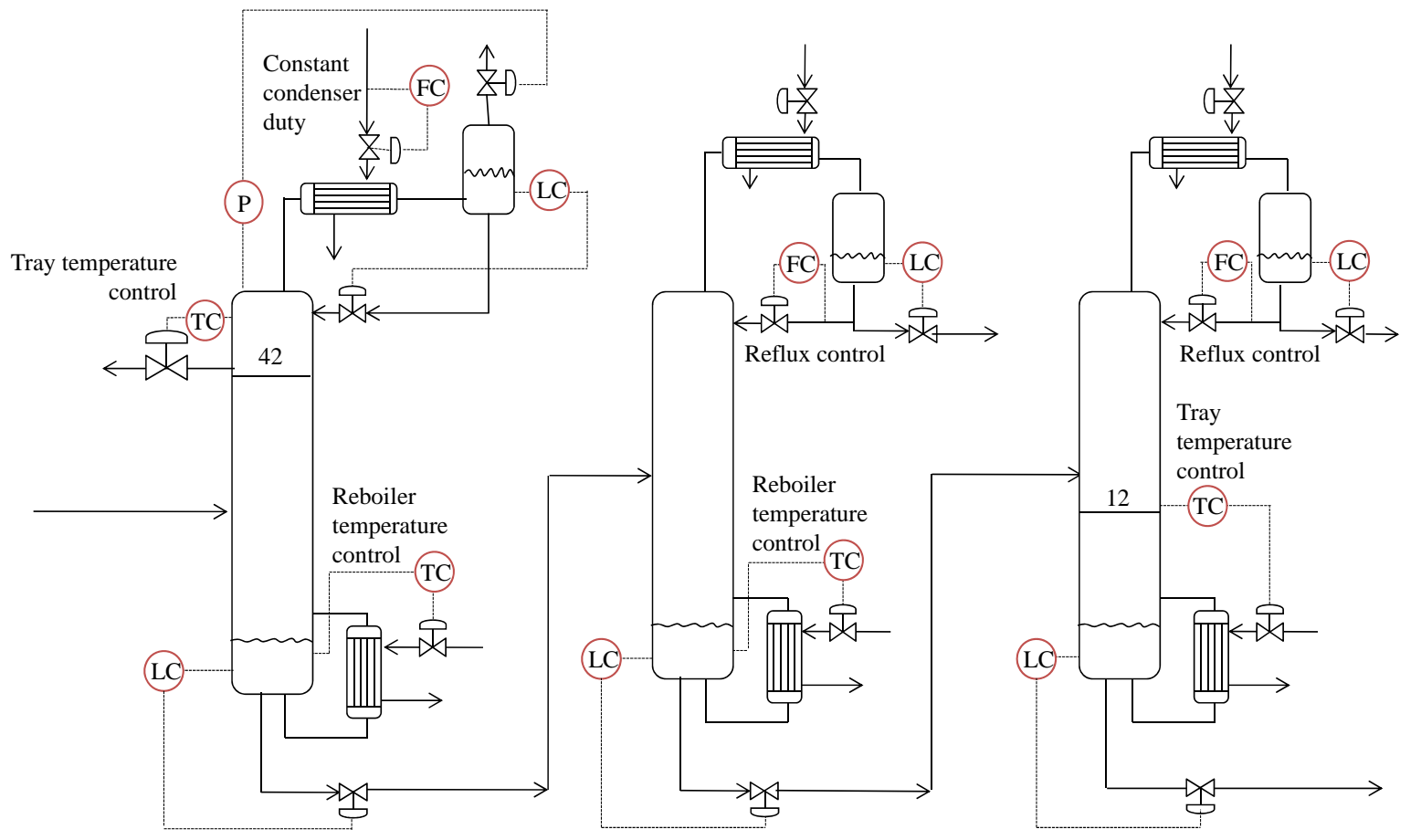

Fig. 8. The base-case control structure

Fig. 9.a. shows the effects of the disturbances scenarios on the temperature profile of the depentanizer distillation column. In this column, heat duty of condenser, temperature of the C5 stream and the temperature of the reboiler are controlled. A comparison of this figure with Fig. 9.a. demonstrates the advantage of the new control structure over the base-case. The required temperature of the condenser in Fig. 9.a. is very low, which shows that in some disturbance scenarios achieving perfect control using the cooling water is impossible. In these scenarios the control valve of the cooling water becomes saturated which results in loss of a control action. 


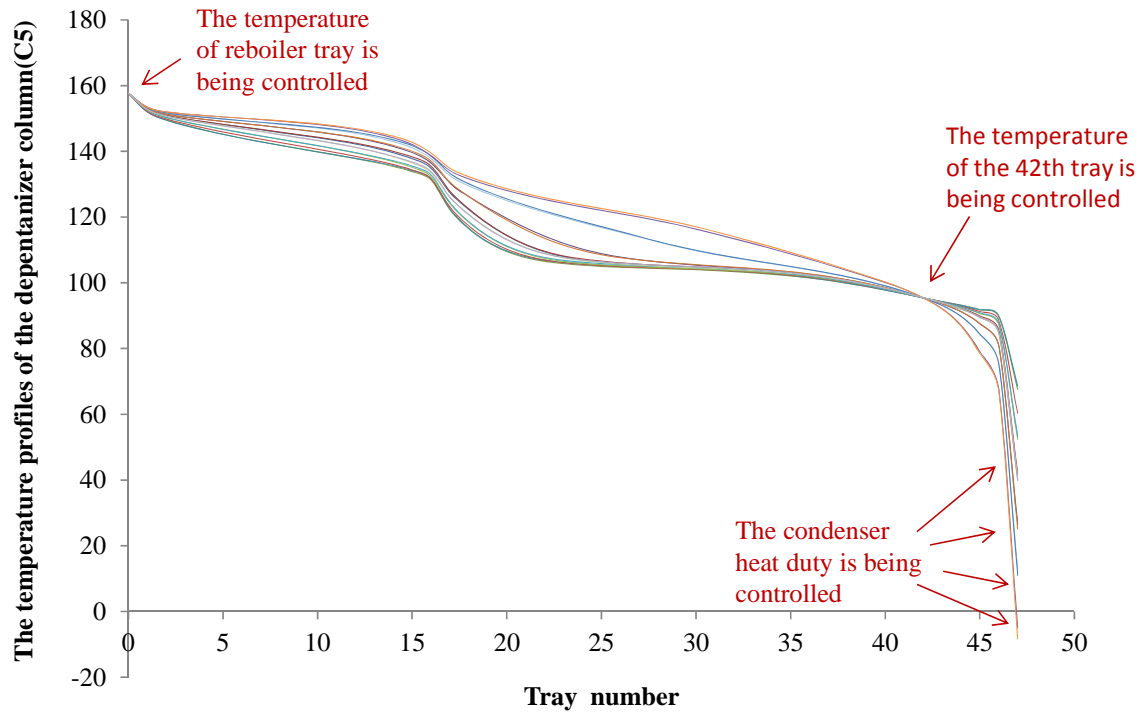

Fig. 9.a. The temperature profiles of the depentanizer column for the sixteen disturbance scenarios for the basecase control structure.

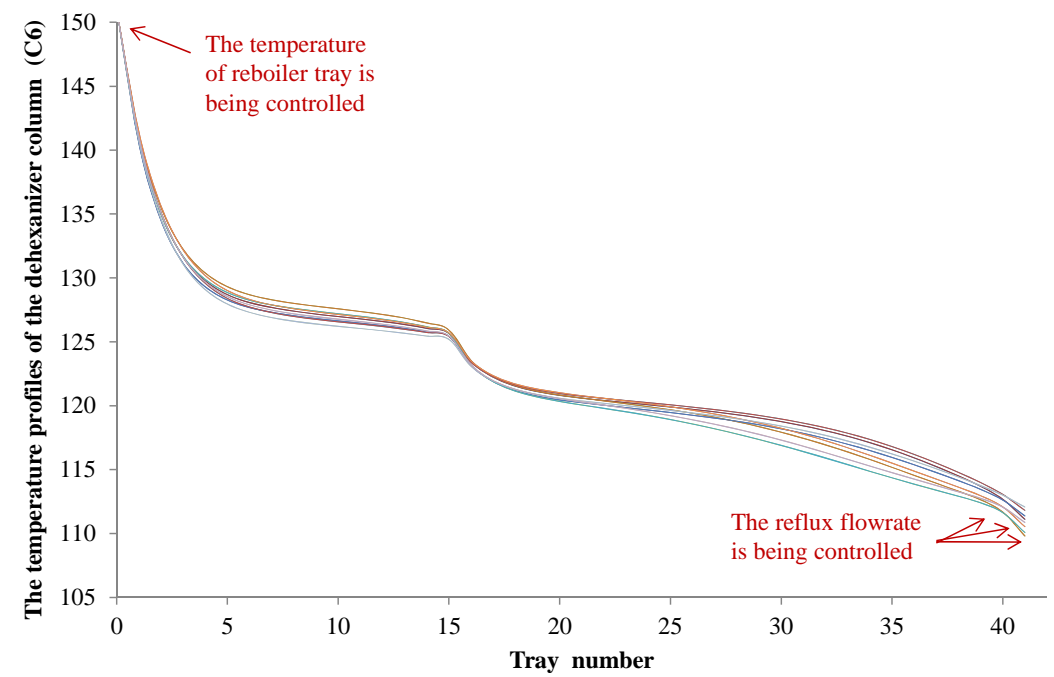

Fig. 9.b. The temperature profiles of the dehexanizer column for the sixteen disturbance scenarios for the basecase control structure.

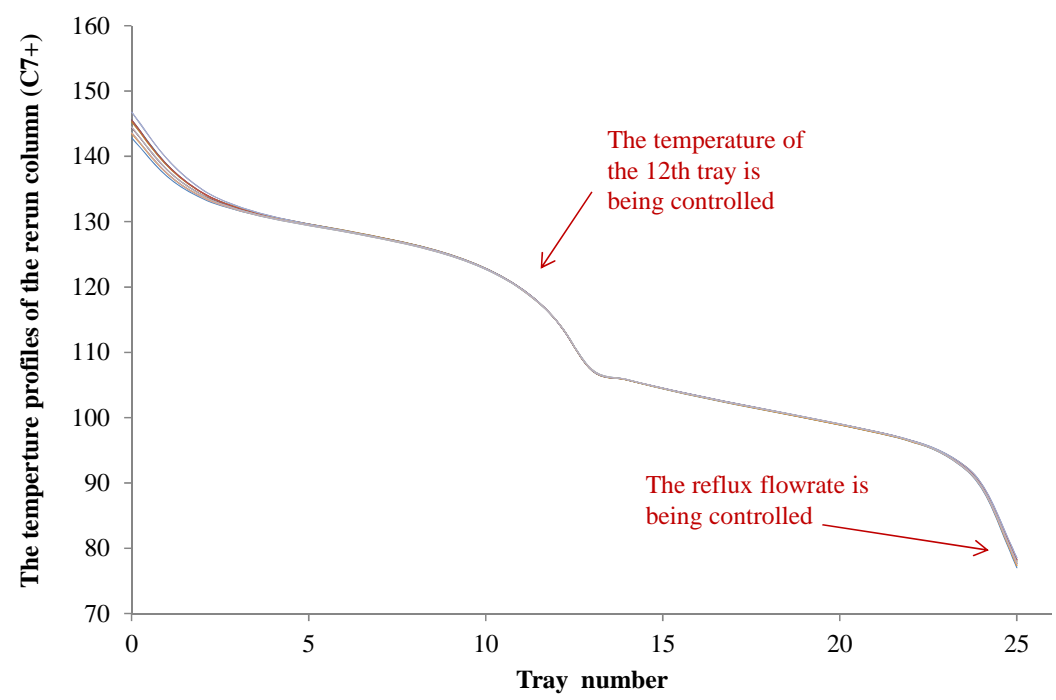

Fig. 9.c. The temperature profiles of the rerun column for the sixteen disturbance scenarios for the base-case control structure. 
Temperature profiles for the dehexanizer and rerun columns are illustrated in Figs. 9.b. and 9.c. which can be compared to Figs. 7.b. and 7.c. In the dehaxanizer column, the reflux flowrate and the temperature of the reboiler are controlled. The task of this column is to separate the product from overheads. A comparison of Fig. 7.b. with Fig 9.b. demonstrates superior performance of the optimized control structure because the product is withdrawn from column overhead and this part of column is less affected in the optimized control structure by disturbances. In the rerun column, the optimized control structure and base-case control structure differ only in the number of the temperature tray. The optimized control structure shows better performance in the column bottom product.

The values of the objective functions of the base-case control structure are compared to the optimized control structure in Table 3. In all objectives, the optimized control structure performs better. However, the optimized control structure manipulates the input variables more. It is because in the base case design, some of the manipulated variables are selected as controlled variables and their flowrates are constant for different disturbance scenarios.

\section{Conclusion}

This article has presented a novel optimization-simulation framework for the optimal selection of control structures. It makes use of the notions of perfect control and inversion of the process model (Garcia 1982). The inverse of the process model represents perfect control, i.e. it outperforms any controller with the same control structure (Yuan 2010). The advantage of this optimization framework is that it postpones the design of controllers and reduces the size of the problem significantly, thus the proposed methodology is scalable and practical for application to larger industrial cases. The proposed framework decomposes the problem into two sub-problems. One sub-problem concerns steady-state controlled variables, the other address the inventory control systems. The second subproblem is addressed using engineering insights and heuristics. Furthermore, the new scheme ensures that the optimized control structure features state controllability. The results of the proposed method in this research demonstrate the benefits of keeping the selected CVs constant and also indicate why one $\mathrm{CV}$ and not another is the best one to use.

The performance of the proposed framework was demonstrated on the case study of an industrial distillation train. The results showed a very good trade-off between different control design objectives, which ensure controllability, and profitability of the synthesized control structure. A comparison of the optimized design and a base case design shows that the optimized design achieves minimum of product loss and ensures that the process is controllable for all disturbance scenarios.

\section{Acknowledgement}

Mahdi Sharifzadeh gratefully acknowledges partial financial support from the 2010 and 2011 ISA Educational Foundation scholarships, the Burkett Scholarship and Ure bursary award of the Department of Chemical Engineering and Chemical Technology, Imperial College London. Nina F. Thornhill gratefully acknowledges the sponsorship of ABB and the Royal Academy of Engineering for the ABB/RAEng Research Chair in Process Automation at Imperial College London.

\section{References}

Aspen-HYSYS document (2009a) Operation guide, Aspen Technology (V7.1).

Aspen-HYSYS document (2009b) Simulation basis, Aspen Technology (V7.1).

Caballero J. A., Odjo A., Grossmann I. E. (2007), Flowsheet optimization with complex cost and size functions using process simulators. AIChE Journal., 53 (9) 2351-2366.

Christofides P. D., Davis, J. F., El-Farra, N. H., Clark D. H., Kevin R. D., Gipson, J. N. (2007). Smart plant operations: Vision, progress and challenges. AIChE Journal 53(11): 2734-2741.

Downs J. J., Skogestad S. (2009). An Industrial and Academic Perspective on Plant-wide Control Annual Reviews in Control 35(1): 99-110.

Downs J. J. (1992). Distillation control in a plantwide control environment. Practical distillation control. W. L. Luyben, (Ed)., Van Nostrand Reinhold, New York, 413-439. 
Edgar T. F., Himmelblau D. M., Lasdon L. S, (2001), Optimization of chemical processes, $2^{\text {nd }}$ edition, McGraw-Hill, Boston.

Edgar T. F. (2004). Control and operations: when does controllability equal profitability? Computers \& Chemical Engineering 29(1): 41-49.

Garcia C. E., Morari Manfred (1982). Internal model control. 1. A unifying review and some new results. Ind. Eng. Chem. Process Des. Dev., 21 (2) 308-323.

Georgakis C., Price R. M. (1993). Plantwide regulatory control design procedure using a tiered framework. Industrial \& Engineering Chemistry Research 32(11): 2693-2705.

Georgakis C., Price, R. M., Lyman, P. R. (1994). Throughput Manipulation in Plantwide Control Structures. Industrial \& Engineering Chemistry Research 33(5): 1197-1207.

Grossmann I. E., Floudas C. A. (1978) Active constraint strategy for flexibility analysis in chemical processes, Computer \& Chemical Engineering, 2(6). 675-693.

Halvorsen I. J., Skogestad S., Morud J. C., Alstad V. (2003). Optimal selection of controlled variables, Industrial \&Engineering Chemistry Research, 42, 3273-3284.

Hori E. S. and Skogestad S. (2007). Selection of control structure and temperature location for twoproduct distillation columns. Chemical Engineering Research and Design 85(3): 293-306.

Jelali M. (2006). An overview of control performance assessment technology and industrial applications. Control Engineering Practice 14(5): 441-466.

Qin S.J., (1998). Control performance monitoring - a review and assessment. Computers \& Chemical Engineering 23(2): 173-186.

Jones D. , Tamiz M. (2010). Practical Goal Programming. Springer, 1st Edition, International Series in Operations Research \& Management Science, Vol. 141.

Kirk-Othmer ( 2004). Encyclopedia of Chemical Technology, Wiley-Interscience. Hoboken, N.J., USA.

Luyben W. L(2004). Chapter A1:The need for simultaneous design education. The Integration of Process Design and Control P. Seferlis M. C. Georgiadis (Ed): 654 Pages. Elsevier, Amsterdam, Netherlands.

Luyben W. L. (2006). Distillation design and control using Aspen simulation. Wiley-Interscience, Hoboken, N.J., USA.

McAvoy, T. J. (1999). Synthesis of Plantwide Control Systems Using Optimization. Industrial \& Engineering Chemistry Research 38(8): 2984-2994.

Mitchell M., (1998), An introduction to genetic algorithms, $1^{\text {st }}$ edition, MIT press, Cambridge, Mass. Narraway, L. T., Perkins J. D. (1993). Selection of process control structure based on linear dynamic economics. Industrial \& Engineering Chemistry Research 32(11): 2681-2692.

Odjo A. O., Sammons N. E., Jr., Yuan W., Marcilla A., Eden M. R., Caballero J. A., (2011), Disjunctive-Genetic Programming Approach to Synthesis of Process Networks, Industrial Engineering and Chemistry Research, 50 (10), 6213-6228, DOI: 10.1021/ie1017699.

Perkins J. D., Wong M. P. F. (1985).Assessing controllability of chemical plants. Chemical Engineering Research and Design 63(6) 358-362.

Perkins J. D., Walsh S. P. K. (1996).Optimization as a tool for design/control integration. Computers \& Chemical Engineering 20(4): 315-323.

Qin S. J., Badgwell T. A. (2003). A survey of industrial model predictive control technology. Control Engineering Practice 11(7): 733-764.

Rawlings J. B., Stewart B. T. (2008). Coordinating multiple optimization-based controllers: New opportunities and challenges. Journal of Process Control 18(9): 839-845.

Rosenbrock H. H. (1970). State-space and multivariable theory. London, Nelson.

Sahinidis V. N., (2004), Optimization under uncertainty: state-of-the-art and opportunities. Computers \& Chemical Engineering, 28 (6-7) 971-983.

Sakizlis V., Perkins J. D., Pistikopoulos E. N. (2004). Recent advances in optimization-based simultaneous process and control design. Computers \& Chemical Engineering 28(10): 2069-2086. Sharifzadeh, M., Rashtchian, D., Pishvaie, M.R., and Thornhill, N.F., (2011), Energy induced separation network synthesis of an olefin compression section: a case study, Industrial Engineering and Chemistry Research, 50, 1610-1623. 
Skogestad S. (2007). The Dos and Don'ts of Distillation Column Control. Chemical Engineering Research and Design 85(1): 13-23.

Stephanopoulos G. , Ng C. (2000). Perspectives on the synthesis of plant-wide control structures. Journal of Process Control 10(2-3): 97-111.

Vasudevan, S., Konda NVSNM, Rangaiah GP, (2009). Plant-wide control: methodologies and applications. Reviews in chemical engineering 25(5-6): 297-337.

Yuan Z., Chen .and Zhao J. (2010). An overview on controllability analysis of chemical processes. AIChE Journal 57(5): 1185-1201. 